\title{
Adaptive Random Re-Routing for Differentiated QoS in Sensor Networks
}

\author{
Erol Gelenbe and Edith C.-H. Ngai \\ Department of Electrical and Electronic Engineering \\ Imperial College London \\ Exhibition Road, South Kensington \\ London SW7 2AZ UK \{e.gelenbe, e.ngai\}@imperial.ac.uk
}

\begin{abstract}
Much of the traffic carried by Sensor Networks will originate from routine measurements or observations by sensors which monitor a particular situation, such as the temperature and humidity in a room or the infrared observation of the perimeter of a house, so that the volume of routine traffic resulting from such observations may be quite high. When important and unusual events occur, such as a sudden fire breaking out or the arrival of an intruder, it will be necessary to convey this new information very urgently through the network to a designated set of sink nodes where this information can be processed and dealt with. This paper addresses the important challenge of avoiding that the volume of routine background traffic creates delays or bottlenecks that impede the rapid delivery of high priority traffic resulting from the unusual events. Specifically we propose a novel technique, the "Randomized Re-Routing Algorithm (RRR)", which detects the presence of novel events in a distributed manner, and dynamically disperses the background traffic towards secondary paths in the network, while creating a "fast track path" which provides better delay and better QoS for the high priority traffic which is carrying the new information. When the surge of new information has subsided, this is again detected by the nodes and they can progressively revert to best QoS or shortest path routing for all the ongoing traffic. The proposed technique is evaluated using a mathematical model as well as simulations.
\end{abstract}

Keywords: Sensor networks, Quality of service, Routing, Performance evaluation, Simulations

\section{INTRODUCTION}

Wireless sensor networks (WSNs), and sensor networks (SNs) in general, must forward significant data promptly and efficiently from the different sensors where the data originates to one or more sinks in the network at which data is collected and where it may also be interpreted. In such networks: (1) routine measurements and sensing take place constantly resulting in a steady volume of data being transmitted towards the sink(s), and (2) unusual events of particular interest will occur unexpectedly, and the information related to such events will require fast transmission to the sink(s). While routine data are essential for reporting on the conditions that the $\mathrm{SN}$ is monitoring, unusual events are more critical and need a faster or "better QoS" treatment by the network, such as short delay, very low loss, possibly high bandwidth, better security, etc..

In this paper we propose an adaptive technique which we call Randomized Re-Routing (RRR) for addressing these needs of SNs based on the following steps:

- During network operation, the network nodes observe the traffic they are conveying and each of them learns the different traffic flows that it may be carrying. Although this paper does not examine the issue of how a packet is actually detected or classified as being high priority, different options can be imagined. In the simplest case, this may just imply that a node maintains the running average value of a measurement which is contained in packets belonging to each of the connections (source-to-destination) that it is conveying, and a packet may be classified as being "normal" if its contents are very similar to those of the running average for the same connection. In other cases, the fact that a previously "quiet" node has suddenly started generating traffic 
may also be considered to be an unusual event. We will assume that a node inserts a ZERO bit header in "normal" packet.

- A node which generates or conveys a packet whose content is considered to be of greater importance, will classify that packet as being "unusual" and insert a ONE bit in its header.

- Each node also monitors the rate at which it receives "unusual" packets, and if this rate does not exceed a threshold $\tau_{0}$, then the node forwards all packets it receives along their preferred (e.g. shortest or best QoS) path towards their destinations. Obviously the preferred path may be determined by criteria such as the minimum delay, greatest security, lowest power consumption, smallest loss etc.

- If a node (source or transit) senses that the rate at which it forwards unusual packets exceeds the threshold $\tau_{0}$, then it will forward all ONE-bit packets along the best QoS path to their destination, while all ZERO-bit carrying packets will be directed along a randomized route which spreads the lower priority traffic across the network away from the high priority paths, reserving the better paths to the high priority traffic.

In the sequel we will present this algorithm in detail and provide an evaluation of its effectiveness using both an analytical model and simulations. In particular, we will use a mathematical approach based on diffusion approximations $[1,2]$ to estimate the resulting packet travel delays from source to destination.

The remainder of the paper is organized as follow. In Section 2, we describe some related work in the area. In Section 3, we discuss our network model for evaluating the travel delay of packets in SNs. In Section 4, we present the RRR algorithm for providing better quality of service to unusual events in SNs. Sections 5 and 6 summarize the numerical and simulation results that we have obtained, and we conclude the paper in Section 7.

\section{RELATED WORK}

Adaptation in packet networks in general is again coming to the forefront [3] and it contributes to the general framework of "autonomic communications" [4, 5]. One significant trend in this work has been to consider routing that can be modified based on on-line measurements of network load so as to respond to quality-of-service needs $[6,7]$.

Several real-time communication protocols have been studied for sensor networks. He et al. [8] propose SPEED, a protocol which combines feedback control and non-deterministic quality of service (QoS) aware geographic forwarding. Lu et al. [9] describe a packet scheduling policy, called Velocity Monotonic Scheduling, which inherently accounts for both time and distance constraints. Felemban et al. [10] propose Multi-path and Multi-Speed Routing Protocol (MMSPEED) for probabilistic QoS guarantee in WSNs. Multiple QoS levels are provided in the timeliness domain by using different delivery speeds, while various requirements are supported by probabilistic multipath forwarding in the reliability domain; our approach has some similarity to this work. Moreover, routing protocols with congestion awareness have also been proposed for ad hoc networks [11, 12].

In our work we focus on the quality of service in forwarding routine data and unusual events in SNs, and consider how to manage routing so that network capacity within the SN is created so as to offer uncongested paths to traffic emanating from unexpected events, in addition to routing the routine parts of the traffic.

Some congestion control algorithms have also been proposed for wireless sensor networks. Wan et al. [13] proposed an energy efficient congestion control scheme for sensor networks called CODA (COngestion Detection and Avoidance), which includes receiver-based congestion detection, open-loop hop-by-hop backpressure, and closed-loop multi-source regulation. Hull et al. [14] have examined three techniques to mitigate congestion in WSN, which includes hop-by-hop flow control, rate limiting source traffic, and prioritized medium access control (MAC). Ee et al. [15] propose a distributed algorithm for congestion control and fairness in many-to-one routing, which measures the average transmission rate, then divides and assigns the average to downstream nodes equally. Some recent work also studies the effect of timeouts on travel delays in wireless sensor networks [16]. 
Most of the existing work requires feedback from sensor nodes which results in extra overhead in the network. In the contrary, the RRR approach we propose is quite simple and easy to implement in a distributed manner since all decisions are locally taken by the nodes, and it requires no feedback messages from the congested nodes.

\section{MATHEMATICAL MODEL}

In this section we present a model of a network which supports sensors distributed over some open or built area. The sensor nodes forward packets containing their measurements or observations towards one or more sink nodes. This may happen at regular intervals or only when certain significant events occur. Since sensor nodes have limited wireless range, so that multi-hop communications are generally required to forward the data to the sinks. We do not make any specific assumptions about the location of the nodes but just consider the distance from hop to hop, and the total distance to the destination node. In order to evaluate the travel delay of packets in the SN, we will use an approach based on diffusion approximations developed in [17, 18, 19], which is also detailed in [20]. This approach was first used to compute travel delays in wireless sensor networks in $[1,2]$ and we briefly recall it in in this section. We model the location of nodes and the relays of packets on the path from source to destination as follows. At time $t$ we assume that a packet is located at some node which is positioned at a distance measured in minimum number of hops $X_{t}$ from the destination. Time changes from hop to hop are represented by an increment of $\Delta t>0$. The value $X_{0}=D$ indicates that the packet is at the source with an initial distance $D$ to the sink at time 0 , while $X_{t}=0$ indicates that the packet is at the sink at time $t$ so that any further transmission of that particular packet ceases. The model allows for the loss of packets, and this is represented by a loss rate (loss per unit time) $f$. Furthermore, we assume that there is some acknowledgement mechanism that informs the source nodes about the correct reception of packets at the sinks; in a wireless sensor network this can be achieved, for instance, via a reserved wireless frequency that the sinks use to broadcast a message saying "packet so-and-so has been received", but it could also be achieved by packets going upstream in a multi-hop manner from the sinks towards the destinations. Let $x$ be the instantaneous value of $X_{t}$, and let $b(x)$ be the average speed at which the packet moves towards the destination at distance $x$ from the sink. Clearly if $b(x)<0$ then the motion is towards the sink, and if $b(x)>0$ then it is drifting away from the sink. Similarly, we have a non-negative second moment parameter $c(x) \geq 0$ which represents the variance of the motion per unit time and is strictly positive if the motion has a random component. If $c(x)=0$ this means that the packet is moving in a deterministic manner. In a well designed system, we would expect that packets carrying data from unusual events would have a negative $b(x)$ and a small $c(x)$, while lower priority routine packets could possibly have a larger value of both $b(x)$ and $c(x)$. Apart from that, $b(x)$ and $c(x)$ also depend on the total traffic arrival rate at the node which is given by $\tau \leq 0$, which is the total rate of arriving packets, except for the packet that is being considered.

\subsection{Delay Computation}

Based on our definitions of $b(x)$ and $c(x)$, in $[1,2]$ the delay $E[T]$ for a packet to travel from its source to destination has been derived as:

$$
E[T]=2 D \frac{1+\frac{f+r}{m}+\frac{f}{r}}{|b|+\sqrt{b^{2}+2 c(f+r)}},
$$

where $D$ is the distance from the source to the destination, and $f \Delta t$ is the probability that the packet is lost in a small time interval $[t, t+\Delta t]$. Since losses can occur, we also assume that a source node use a time-out of average value $1 / r$ to decide when to retransmit a packet, and that after the time-out it actually waits another $1 / \mathrm{m}$ time units on the average before actually retransmitting the packet that is assumed to have been lost. Note that the time-out will operate both when the packet is actually lost, and when the packet's travel time has exceeded the time-out delay. The mathematical model assumes that both the time-out and the additional "safety time" are exponentially distributed. 


\section{RANDOMIZED RE-ROUTING (RRR)}

In order to provide preferential treatment to ONE-bit packets, the RRR algorithm routes the two types of packets differently. More specifically, packets from unusual events are routed along the shortest paths, while the routine data are randomly shunted to slower secondary path.

The RRR algorithm operates in the following manner at any source or intermediate node:

- If at time $t$ the node locally observes a level of arriving traffic $\tau_{t}$ which is below a given threshold $\tau_{0}$, then traditional geographic routing [21] is applied to all packets.

- However, if $\tau_{t}>\tau_{0}$, then ZERO-bit packets and ONE-bit packets, whether they arrive from some other node for forwarding, or are generated internally, will be routed differently as follows.

- Each node $i$ ranks its neighboring nodes $i_{1}, . . i_{H}$ so that $i_{1}$ is located closest to the sink in number of hops, and $i_{H}$ is the one which is farthest away. Node $i$ forwards ONE-bit packets to neighbors $i_{1}, \ldots, i_{K}$, and

- The node forwards all ZERO-bit packets to the remaining neighbors $i_{K+1}, \ldots, i_{H}$. Note that in general we will select one of these output nodes at random among the given set, and also we may choose not to use some of the nodes at the tail end of the ranking, because they may lead to excessively long paths.

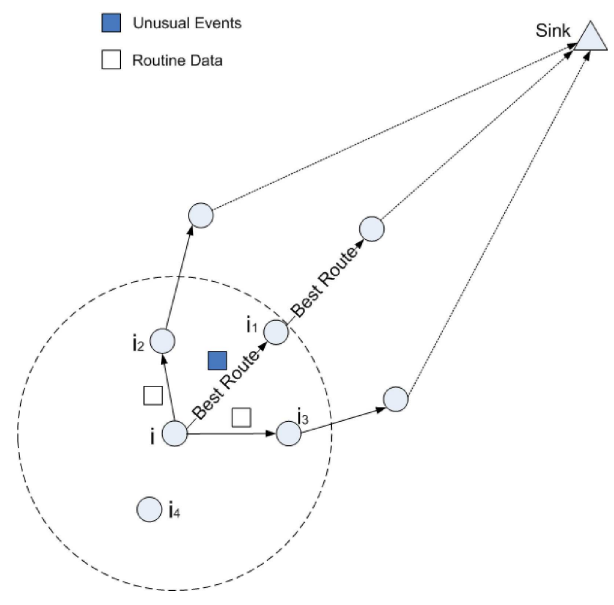

FIGURE 1: Randomized re-routing for routine data and unusual events.

Figure 1 illustrates how the RRR algorithm operates. Here node $i$ has four neighbors $i_{1}, i_{2}, i_{3}$, and $i_{4}$ which are ordered according to their distances to the sink. When $i$ receives a ONE-bit packet and forwards it to its neighbor which is closest to the destination, while it forwards ZERO-bit packets to $i_{2}$ and $i_{3}$ with equal probability. Note that $i_{4}$ is not chosen as it is located further away from the sink than $i$ itself. From this example, we see that our algorithm allocates the best route for transmitting important event data, while routine data are pushed aside to the remaining routes to achieve quality of service.

\section{PERFORMANCE ANALYSIS BASED ON THE DIFFUSION MODEL}

Our evaluation includes both a theoretical component and simulations. We will present some additional analytical results here, followed by the simulation results in the next section.

Consider an example with $D=10, m=0.02, \mu=30 \mathrm{pkt} / \mathrm{s}, \tau=5 \mathrm{pkt} / \mathrm{s}$ and let us compute the travel delay of packets in a network with unusual events. When unusual events occur, network traffic increases suddenly and RRR is applied. The ONE-bit packets are routed along the shortest paths, while ZERO-bit packets are routed probabilistically along the remaining paths.

Define the advancement vector $H=[-1,-1,0,0,1,1]$, for a node with six neighbors, where the first two are one hop closer to the destination, two are at the same distance, and two are one hop further 
away from the sink than the current node. We use probability vectors for the direction taken by ONE-bit and ZERO-bit packets as $P_{U}=[1,0,0,0,0,0]$ and $P_{R}=[0,1 / 3,1 / 3,1 / 3,0,0]$, respectively, indicating that ONE-bit packets are routed to the neighbor that is one hop closer to the sink, while the ZERO-bit packets are routed to the neighbors with $-1,0$, and 0 hops of advancement with equal probability $1 / 3$.

Figure 2 shows the travel delay of RRR after unusual events occur. The travel delay of ONE-bit packets is much lower than that of the ZERO-bit packets. Similarly, Figure 3 shows the travel delay of packets in RRR with $D=10, m=0.02, \mu=50 \mathrm{pkt} / \mathrm{s}, \tau=10 \mathrm{pkt} / \mathrm{s}$. Again, it shows that ONE-bit packets achieve shorter travel delay than ZERO-bit packets. We increase the service rate to $\mu=500 \mathrm{pkt} / \mathrm{s}$.

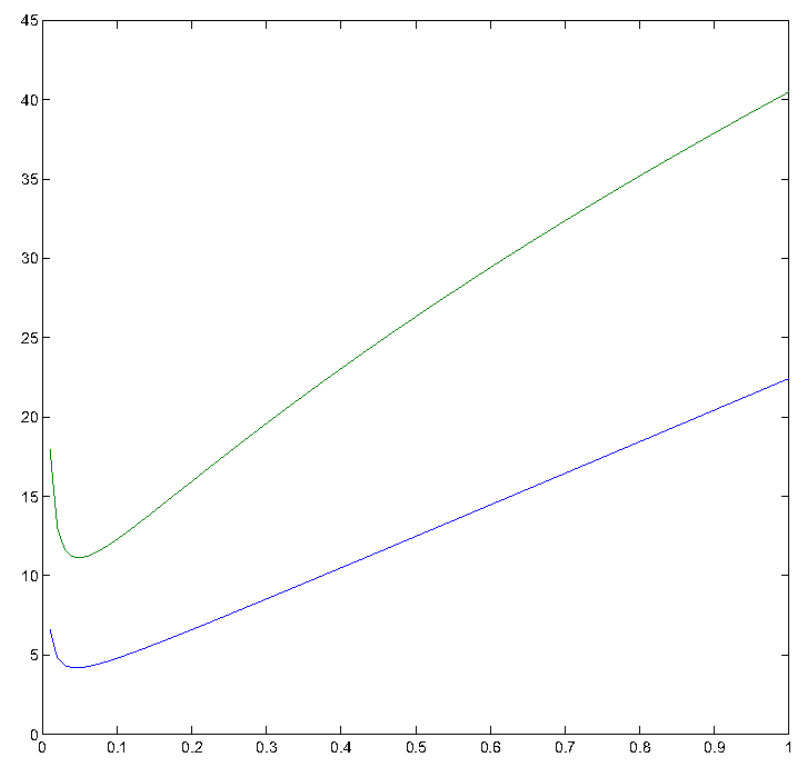

FIGURE 2: After unusual events occur, transmission delay with $f=0.1, D=10, m=0.02, \mu=30 \mathrm{pkt} / \mathrm{s}, \tau=5 \mathrm{pkt} / \mathrm{s}$.

\subsection{The traffic arrival rate $\tau$ at nodes}

Even though RRR can provide better QoS to ONE-bit packets at the cost of lower QoS for the remaining packets, it does have the drawback of potentially increasing the total average arrival rate of packets as a whole at each node because ZERO-bit packets will visit more nodes than if they had taken the shortest path.

$\tau$ the total traffic arrival rate of packets per node depends on the packet arrival rates of unusual and routine packets. If the network as a whole has $n$ nodes and there are $s_{R}$ nodes that are sources of routine data at rate $\lambda_{R}$, with an average number of hops to the sink of $d_{R}$, the total average ZERO-bit traffic generated per node is then

$$
\tau_{R}=\frac{s_{R} * d_{R} * \lambda_{R}}{n}
$$

Now if there are $s_{U}$ nodes which are sources of unusual events each generating $\lambda_{U}$ packets per second, and if they travel on the average $d_{U}$ hops to the destination, they will now generate an additional average traffic rate per node of

$$
\tau_{U}=\frac{s_{U} * d_{U} * \lambda_{U}}{n}
$$

Both $d_{R}$ and $d_{U}$ also depend on the loss probability and other parameters such as the time-out.

The average incoming traffic rate per node is then

$$
\tau=\tau_{R}+\tau_{U}
$$

over the set of all $n$ nodes in the network. Since $d_{R}$ increases when RRR is used, it follows that all packets may experience greater delays per node when RRR is used. 


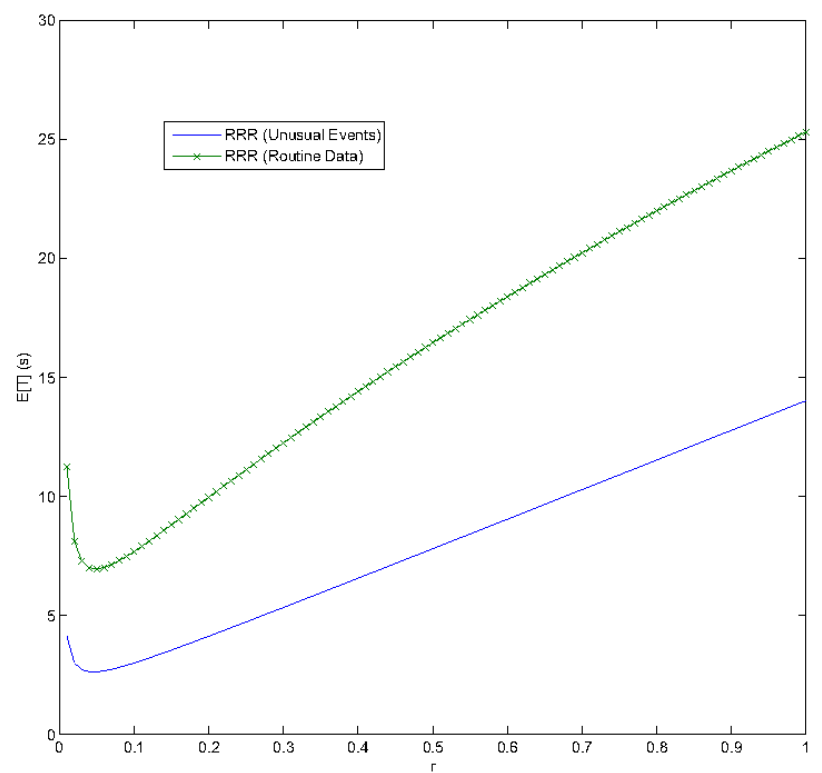

FIGURE 3: After unusual events occur, transmission delay with $f=0.1, D=10, m=0.02, \mu=50 \mathrm{pkt} / \mathrm{s}$, $\tau=10 \mathrm{pkt} / \mathrm{s}$.

However the preceding analysis is based on averages over the whole network, and RRR will in practice reduce the traffic experienced by nodes which carry ONE-bit traffic while increasing the traffic at nodes that are used by ZERO-bit packets.

\subsection{Travel delay for the RRR algorithm}

Using the vectors $H$ and $P$ defined in Section 5, we can compute the $b_{U}$ parameter for the travel delay (in number of hops) for the ONE-bit packets as:

$$
b_{U}=\sum_{j} P_{j}^{U} H_{j}
$$

where $P_{j}^{U}$ is the probability that a node selects the $j$-th next neighbor as next hop to forward a ONE-bit packet. Similarly,

$$
\begin{aligned}
c_{U} & =\sum_{j} P_{j}^{U} H_{j}^{2}-\left(b_{U}\right)^{2} \\
& =\sum_{j} P_{j}^{U} H_{j}^{2}-\left(\sum_{j} P_{j}^{U} H_{j}\right)^{2}
\end{aligned}
$$

and the diffusion model will yield $d_{U}$ the average number of hops for ONE-bit packets to reach a destination which is at distance $D$ hops.

In order to consider the total travel delay, we also have to compute the average delay $Q$ (queueing plus transmission) through each hop for each type of traffic which we will approximate using a $M / M / 1$ queue yielding:

$$
\overline{Q_{U}}=\frac{\mu^{-1}}{1-\rho_{U}},
$$

where $\rho_{U}=\tau_{U} / \mu$ and $1 / \mu$ is the link transmission delay per packet plus any processing delay through a node.

The total source-destination travel delay of the $U$ packets is then

$$
E\left[T_{U}\right]=\left[\frac{2 D \mu^{-1}}{1-\frac{\tau_{U}}{\mu}}\right] \frac{1+\frac{f+r}{m}+\frac{f}{r}}{\left|b_{U}\right|+\sqrt{b_{U}^{2}+2 c_{U}(f+r)}} .
$$


When forwarding ZERO-bit packets, nodes have a different set of probabilities for selecting neighbors as discussed in Section 5. Using a similar approach as in the previous paragraph we will get:

$$
b_{R}=\sum_{j} P_{j}^{R} H_{j}
$$

and

$$
\begin{aligned}
c_{R} & =\sum_{j} P_{j}^{R} H_{j}^{2}-b_{R}^{2} \\
& =\sum_{j} P_{j}^{R} H_{j}^{2}-\left(\sum_{j} P_{j}^{R} H_{j}\right)^{2}
\end{aligned}
$$

so that the total average travel delay for ZERO-bit packets which originate at distance $D$ hops from their destination is:

$$
E\left[T_{R}\right]=\left[\frac{2 D \mu^{-1}}{1-\frac{\tau_{R}}{\mu}}\right] \frac{1+\frac{f+r}{m}+\frac{f}{r}}{\left|b_{R}\right|+\sqrt{b_{R}^{2}+2 c_{R}(f+r)}} .
$$

Note that we have assumed that $\tau$ is the same for all nodes, which is a worst case assumption for the nodes carrying ONE-bit packets.

\subsubsection{Figures of Merit}

In order to evaluate the effectiveness of the RRR algorithm, we consider two figures of merit. The first figure evaluates the preferential treatment of ONE-bit packets with respect to ZERO-bit packets, when both types of packets originate from nodes situated at the same distance to the destination.

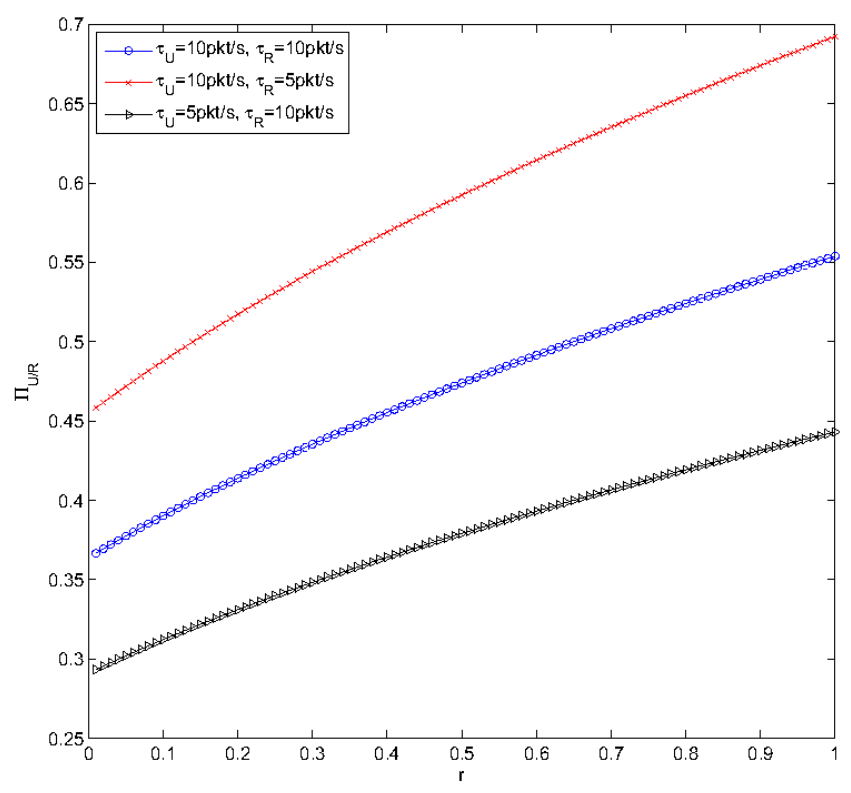

FIGURE 4: Ratio $\Pi_{U / R}$ with $\mu=30 \mathrm{pkt} / \mathrm{s}$ showing merit of the RRR algorithm.

Figure 4 shows the ratio $\Pi_{U / R}=E\left[T_{U}\right] / E\left[T_{R}\right]$ which is:

$$
\Pi_{U / R}=\frac{1-\frac{\tau_{R}}{\mu}}{1-\frac{\tau_{U}}{\mu}} \frac{\left|b_{R}\right|+\sqrt{b_{R}^{2}+2 c_{R}(f+r)}}{\left|b_{U}\right|+\sqrt{b_{U}^{2}+2 c_{U}(f+r)}},
$$

where it is assumed that with RRR distinct nodes carry different types of packets are distinct.

We then compare the effect on ONE-bit packets of the use of the RRR algorithm, against the case where all packets are treated in the same manner in Figure 5. In the latter case, we will assume that 


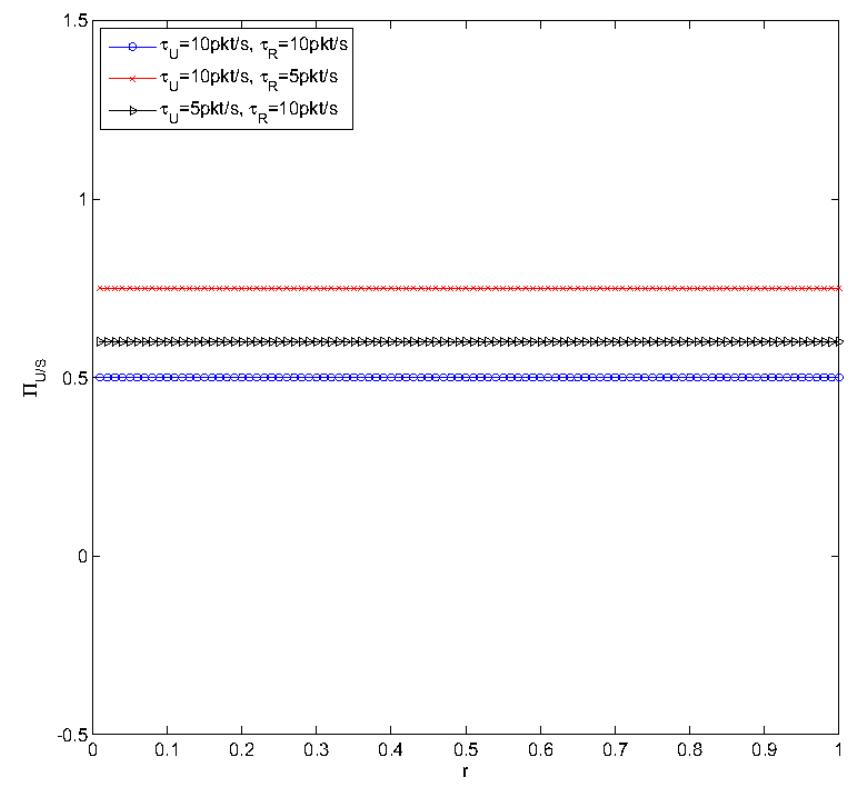

FIGURE 5: Ratio $\Pi_{U / S}$ with $\mu=30 \mathrm{pkt} / \mathrm{s}, b=-1$ and $c=0$.

all packets take the shortest route to the destination $d=d_{R}$ so that the average traffic arrival rate to all nodes is $\tau_{S}$ given by:

$$
\tau \geq \tau_{S}=\frac{\left(s_{R} \lambda_{R}+s_{U} \lambda_{U}\right) d}{n}
$$

while $b=-1$ and $c=0$ since all packets select the shortest path with no randomness. The figure of merit then becomes $\Pi_{U / S}=E\left[T_{U}\right] / E\left[T_{S}\right]$ where

$$
E\left[T_{S}\right]=\left[\frac{D \mu^{-1}}{1-\frac{\tau_{S}}{\mu}}\right] \cdot\left[1+\frac{f+r}{m}+\frac{f}{r}\right] .
$$

$E\left[T_{S}\right]$ is the packet travel delay of ONE-bit packets in RRR algorithm, while $E\left[T_{R}\right]$ is the packet travel delay of ONE-bit packets in traditional geographical routing which use shortest paths for all ONE-bit and ZERO-bit packets.

If ONE-bit packets in RRR also take the shortest path in a deterministic fashion, such that $b_{U}=-1$ and $c_{U}=0$ as well, then:

$$
\Pi_{U / S}=\frac{1-\frac{\tau_{S}}{\mu}}{1-\frac{\tau_{U}}{\mu}},
$$

or

$$
\Pi_{U / S}=\frac{\frac{n \mu}{d}-\lambda_{R} s_{R}-\lambda_{U} s_{U}}{\frac{n \mu}{d}-\lambda_{U} s_{U}} .
$$

Note also that it may be reasonable to take $d \approx \log n$.

\section{SIMULATIONS OF THE RRR ALGORITHM}

We have conducted extensive simulations using the $n s-2$ tool [22] to evaluate the RRR algorithm. The simulation parameters that we have chosen selected so as to be compatible with other studies of SNs $[8,10,23]$. We focus on a WSN which collects and reports routine data to the sink constantly. Any of the sensors has a probability $p$ to be the source of routine data and generates data independently of the other nodes; note that this independence assumption may be unreasonable when correlated events are being reported across a sensory field. Under normal conditions the sensors they report routine data to the sink at a low data rate. Unusual events are assumed to occur infrequently, and in the simulations we have included four nodes which simulate the sources of such events which generate a high traffic rate. We have also introduced a probability of packet loss at each node given by the parameter $f$. 


\subsection{The effect of unusual events}

In the first experiment we have run, there are a total of 100 sensors, each of which is uniformly distributed in an area whose size is $200 \times 200$ meters. A single sink is located the center position $(100,100)$ of the area.

When the simulation begins, only routine data is generated and after 1800 seconds four nodes located at $(50,50),(50,150),(150,50)$ and $(150,150)$ start generating the unusual traffic. We set $p=0.5$ and $f=0$. The data rates of routine data and unusual events are $\lambda_{R}=1 \mathrm{pkt} / \mathrm{s}$ and $\lambda_{U}=5 \mathrm{pkt} / \mathrm{s}$, respectively. Figure 6 shows how the travel delay of packets changes with time. When there are no unusual events, the travel delay of unusual events. The travel delay of packets emanating from four routine data sources located at $(30,100),(170,100)$ and $(100,30),(100,170)$, and hence located at the same distance to the sink as the unusual events, is also plotted for comparison.

When the simulation begins, each node is forwarding its packets to a set of neighbors with equal probability as if the network had in the past experienced some unusual events. After some time, the nodes switch back to shortest path geographic routing for the routine data packets since they have learned that no unusual event data packets are arriving to them, and the travel delay of routine data drops significantly. The travel delay of the routine packets from the four reference source nodes is lower than the overall average since the reference nodes are closer to the sink than other nodes on average. When the unusual events occur at time $1100 \mathrm{~s}$, RRR starts operating. The ONE-bit packets are routed along the shortest paths while the ZERO-bit packets, which are still generated at the same rate, use the randomized routing scheme and hence suffer higher delay.

Figure 7 shows the results of the same experiment with another set of reference points of routine data sources at $(30,30),(30,170),(170,30),(170,170)$. Again, it shows that our proposed RRR algorithm yields the results we expect both in terms of adaptation and in providing far better travel delays to the ONE-bit packets. Since in this case the reference points of the routine data sources are located further away from the sink than the "average node", their travel delays are higher than the average delay.
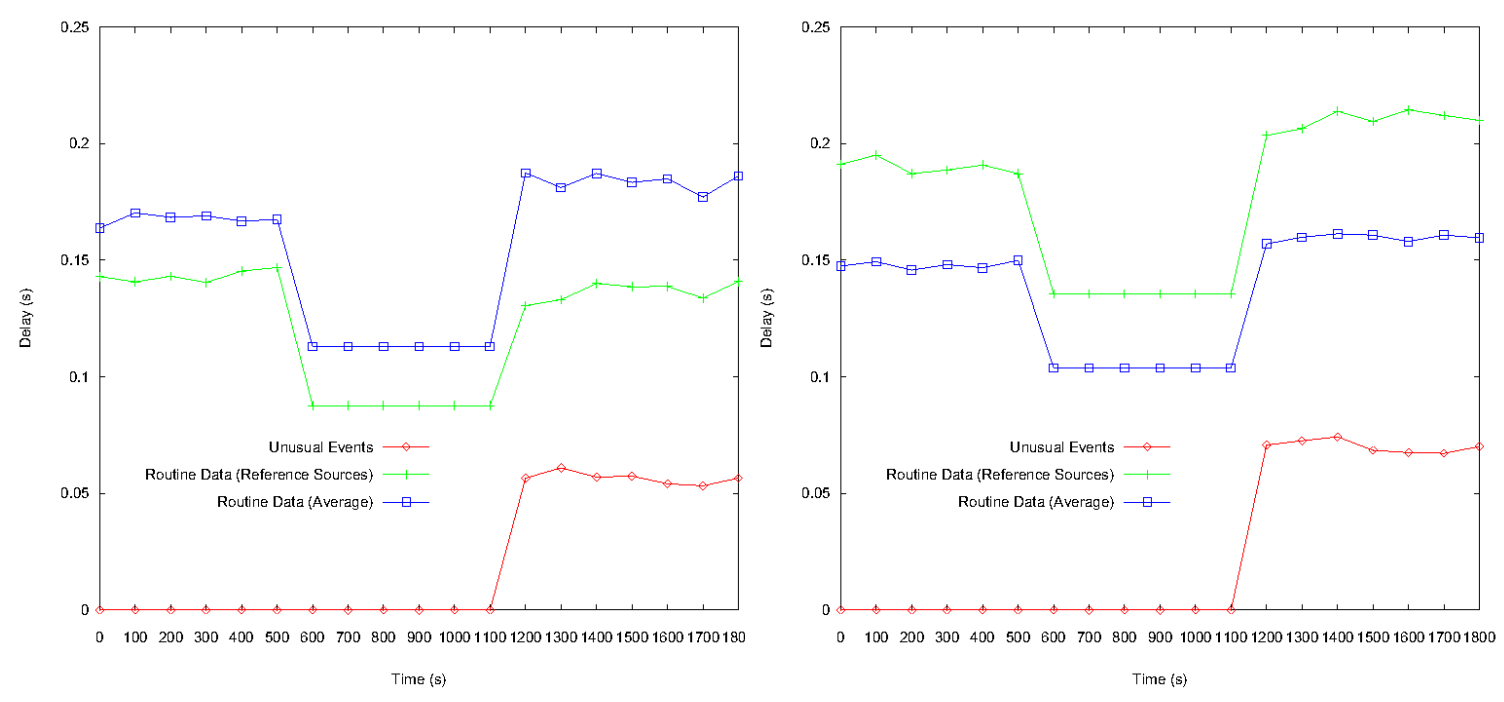

FIGURE 6: Travel delay in three phases with $f=0$, FIGURE 7: Travel delay in three phases with $f=0$, $\lambda_{U}=5 \mathrm{pkt} / \mathrm{s}, \lambda_{R}=1 \mathrm{pkt} / \mathrm{s}$, reference routine data sources $\lambda_{U}=5 \mathrm{pkt} / \mathrm{s}, \lambda_{R}=1 \mathrm{pkt} / \mathrm{s}$, reference routine data sources at $(30,100),(170,100),(100,30)$, and $(100,170)$ at $(30,30),(30,170),(170,30),(170,170)$.

\subsection{Effect of $1 / r$}

In the next experiments, we consider a larger network with 400 nodes deployed in an area of size $400 m \times 400$ meters with the sink at its centre. The network includes packet losses with $f=0.1$. The source nodes incorporate a time-out mechanism to retransmit the packet if it does not receive an acknowledgement from the receiver by a certain time while a packet within the network will be 
destroyed if it has travelled for more than that time. After the time-out, the source node retransmits the packet after an additional delay $M$. Note that in the mathematical models of the previous sections, the time out and additional delay have been assumed to be exponentially distributed random variables. However, we have taken both of these quantities to be constants in our simulations with values $1 / r$ and $1 / m$ respectively.

Figure 8 shows the travel delay $E[T]$ of packets with $p=0.2, M=0.02 s, \lambda_{U}=5 p k t / s$, and $\lambda_{R}=1 \mathrm{pkt} / \mathrm{s}$. The figure shows the same general form of curve as in the analytical results of the previous section, and there is indeed an optimum value of $E[T]$ in both cases. When the time-out value is small, $E[T]$ is extremely high. When it is greater than the optimal value, $E[T]$ increases again. The travel delay for ONE-bit packets is clearly shorter than that of the routine data packets.

Figure 9 illustrates the results of the same experiment with $p=0.4$ and the travel delay here is greater than that in Figure 8.
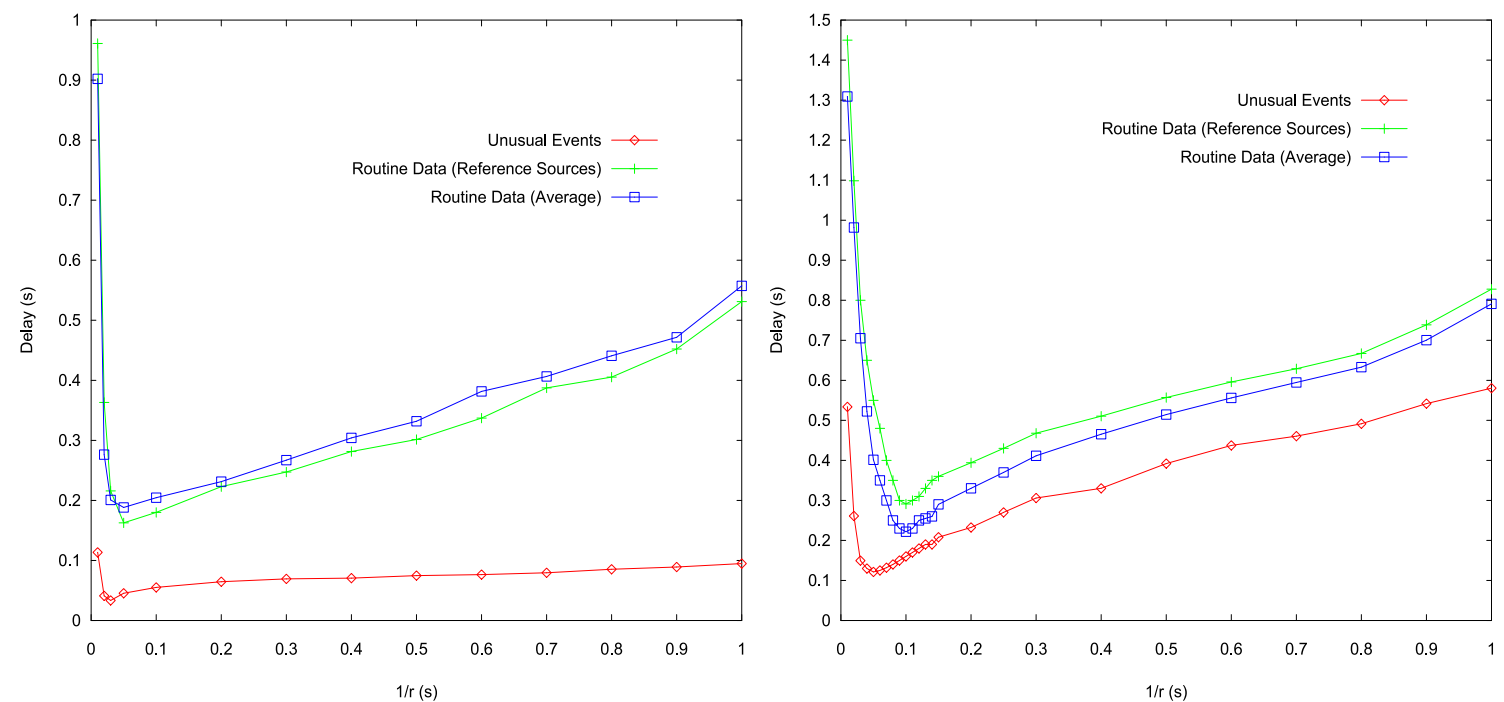

FIGURE 8: Travel delay versus $r^{-1}$ with $f=0.1, p=0.2$, FIGURE 9: Travel delay versus $r^{-1}$ with $f=0, p=0.4$, $m=0.02, \lambda_{U}=5 \mathrm{pkt} / \mathrm{s}, \lambda_{R}=1 \mathrm{pkt} / \mathrm{s}$. $m=0.02, \lambda_{U}=5 \mathrm{pkt} / \mathrm{s}, \lambda_{R}=1 \mathrm{pkt} / \mathrm{s}$.

\subsection{Comparison with geographic routing}

In this section we use simulations to compare RRR with GPSR (Greedy Perimeter Stateless Routing), which is a traditional geographic routing algorithm that forwards packets to the neighbor that is closest to the destination [21]. We place two sources of unusual events and four sources of routine data at the left bottom corner of the network.

Figure 10 shows the travel delay of packets as the time-out delay is varied. We see that RRR achieves lower travel delay for ONE-bit packets than does GPSR. The reason is that RRR utilizes the network capacity more effectively by making use of the nodes that were not used previously, so that the traffic on the busiest paths can be reduced. The travel delay of ONE-bit packets in our routing algorithm is also much lower than that ZERO-bit packets, while the travel delay of both usual and unusual event packets are almost the same in GPSR as all packets routed along the shortest paths. Note that the small differences in average delay observed with GPSR for routine and unusual events is simply due to the fact that in the simulation, the corresponding source nodes in either case have distinct locations.

Even though routine data packets suffer higher transmission delay in RRR due to their randomized routing, the delay achieved is still comparable with GPSR. The reason for this appears to be that RRR has the advantage of distributing ZERO-bit traffic evenly on different paths, thus reducing congestion on all paths. This shows that the RRR algorithms is a very simple and effective method tool for achieving quality of service in the presence of low and high priority traffic streams. 


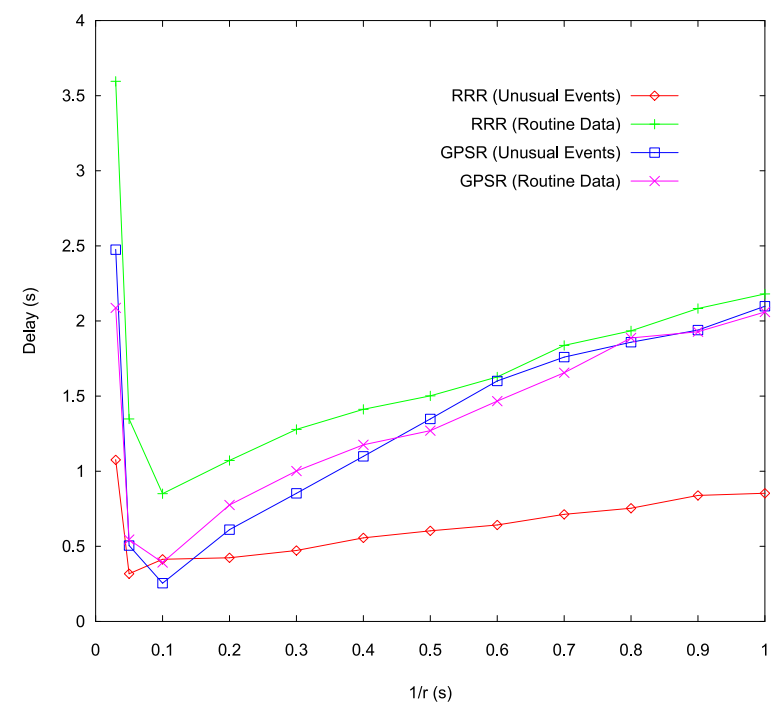

FIGURE 10: Travel delay with $f=0.1 \mathrm{~m}=0.02, \lambda_{U}=10 \mathrm{pkt} / \mathrm{s}, \lambda_{R}=10 \mathrm{pkt} / \mathrm{s}$.

\section{CONCLUSIONS}

In this paper we have proposed a simple randomized algorithm that is designed to react to congestion caused by unusual events in SNs so as to provide better quality of service to the packets carrying the novel or unusual data.

We have evaluated the RRR algorithm using a theoretical model based on diffusion approximations, and also presented numerous simulations. Both the analysis and the simulations show that RRR can achieve significant QoS improvements for high priority traffic, while offering acceptable QoS levels to secondary traffic streams. When unusual events occur in the network, RRR has the added advantage of distributing the "routine" traffic streams randomly over secondary paths in the network so as to reduce congestion on the more heavily used shortest paths.

\section{REFERENCES}

[1] Gelenbe, E. (2006) Travel delay in a large wireless ad hoc network. Proc. of 2nd Workshop on Spatial Stochastic Modeling of Wireless Networks, Boston, April.

[2] Gelenbe, E. (2007) A diffusion model for packet travel time in a random mult-hop medium. ACM Trans. on Sensor Networks, 2.

[3] Gelenbe, E. (2005) Users and services in intelligent networks. Lecture Notes in Computer Science, December, pp. 30-45. Invited paper. Cho, Kenjiro; Jacquet, Philippe (Eds.).

[4] Gelenbe, E., Lent, R., and Xu, Z. (2001) Design and performance of cognitive packet networks. Perform. Eval., 46, 155-176.

[5] Gelenbe, E., Lent, R., Montuori, A., and Xu, Z. (2002) Cognitive packet networks: QoS and performance. Proc. of the IEEE MASCOTS Conference, Ft. Worth, TX, October, pp. 3-12. Opening Keynote Paper.

[6] Gelenbe, E., Lent, R., and Xu, Z. (2001) Measurement and performance of a cognitive packet network. Journal of Computer Networks, 37, 691-701.

[7] Gelenbe, E. (2003) Sensible decisions based on QoS. Computational Management Science, 1, $1-14$.

[8] He, T., Stankovic, J., Lu, C., and Abdelzaher, T. (2003) SPEED: a real-time routing protocol for sensor networks. Proc. of IEEE ICDCS, Providence, RI, U.S., May, pp. 46-55.

[9] Lu, C., Blum, B. M., Abdelzaher, T. F., Stankovic, J. A., and He, T. (2002) RAP: a real-time communication architecture for large-scale wireless sensor networks. Proc. of IEEE RTAS, San Jose, CA, U.S., Sep.

[10] Felemban, E., Lee, C.-G., and Ekici, E. (2006) MMSPEED: multipath multi-speed protocol for QoS guarantee of reliability and timeliness in wireless sensor networks. IEEE Trans. on Mobile 
Computing, 5, 738-754.

[11] Tran, D. and Raghavendra, H. (2005) Routing with congestion awareness and adaptivity in mobile ad hoc networks. Proc. of IEEE WCNC, Mar.

[12] Sankarasubramaniam, Y., Özgür B. Akan, and Akyildiz, I. F. (2003) ESRT: event-to-sink reliable transport in wireless sensor networks. Proc. of ACM Mobihoc, pp. 177-188.

[13] Wan, C.-Y., Eisenman, S. B., and Campbell, A. T. (2003) CODA: congestion detection and avoidance in sensor networks. Proc. of ACM SenSys, Los Angeles, California, U.S., Nov, pp. 266-279.

[14] Hull, B., Jamieson, K., and Balakrishnan, H. (2004) Mitigating congestion in wireless sensor networks. Proc. of ACM SenSys, Baltimore, Maryland, U.S., Nov, pp. 134-147.

[15] Ee, C. T. and Bajcsy, R. (2004) Congestion control and fairness for many-to-one routing in sensor networks. Proc. of ACM SenSys, Baltimore, Maryland, U.S., Nov.

[16] Gelenbe, E., Filippoupolitis, A., and Eid, I. (2007) Energy and time trade-offs in duplicate packet transmission. Proc. of SPECTS 2007, July.

[17] Gelenbe, E. (1975) On approximate computer system models. Journal ACM, 22, 261-269.

[18] Gelenbe, E. and Pujolle, G. (1976) An approximation to the behaviour of a single queue in a network. Acta Informatica, 7, 123-136.

[19] Adams, C., Gelenbe, E., and Vicard, J. (1979) An experimentally validated model of the paging drum. Acta Informatica, 11, 103-117.

[20] Gelenbe, E. and Mitrani, I. (1980) Analysis and synthesis of computer systems. Academic Press (London and New York), ?

[21] Karp, B. and Kung, H. (2000) GPSR: Greedy perimeter stateless routing for wireless networks. Proc. of ACM Mobicom, Boston, Massachusetts, U.S.

[22] Fall, K. and Varadhan, K. (2003) The ns manual. http://www.isi.edu/nsnam/ns.

[23] Ngai, E. C.-H., Zhou, Y., Lyu, M. R., and Liu, J. (2006) Reliable reporting of delay-sensitive events in wireless sensor-actuator networks. Proc. of IEEE MASS, Vancouver, Canada, Oct. 